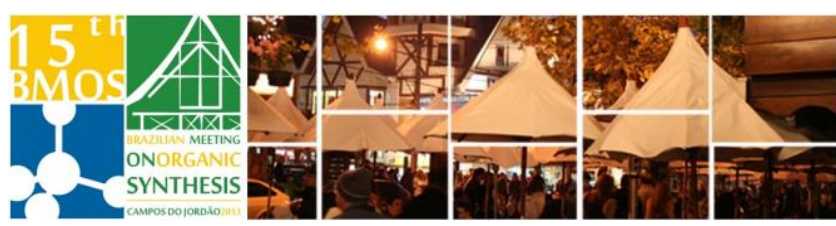

\title{
Development of Methodology for Glycopeptide Enrichment
}

\author{
Caixeta, Vanessa and Westerlind ${ }^{\star}$, Ulrika \\ Leibniz-Institut für Analytische Wissenschaften - ISAS - e.V. \\ Otto-Hahn-Straße 6b, 44227, Dortmund, Germany \\ *ulrika.westerlind@isas.de
}

Keywords: glycoprotein, enrichment method, labeling

\section{INTRODUCTION}

Glycosylation is one of the most common posttranslational modifications, found on more than $50 \%$ of proteins. The majority of glycoproteins are involved in protein-protein interactions on the extra cellular surface that influence growth factors; cellular communication; hormone activities; immunological evasion and tumor progression as examples. ${ }^{1}$

Due to its importance, glycoproteins are a potential source for biomarker discovery in order to detect diseases at early stage and to increase patience surveillance. It is known that disease progression is often related to pathway dysregulation including changes of the glycosylation extension as well as the carbohydrate structure. ${ }^{2}$

Nowadays, the strategy adopted for glycoproteins studies comprises enrichment and standard proteomic analysis using mass spectrometry (MS). However using this approach, it is obtained a small quantity of peptides containing the glyco motif compared to the total amount of peptides which makes the glycopeptides characterization difficult. For this reason it is important to propose methodologies to enrich glycopeptides or glycoproteins from others.

\section{RESULTS AND DISCUSSION}

A method for glycoprotein enrichment is through introduction of bioorthogonal functional groups. Aldehydes react with hydroxylamine compounds to form an oxime linkage under physiological conditions. ${ }^{3}$

We have improved the methodology for oxime ligation enrichment of glycopeptides/glycoproteins by introducing a cleavable linker on magnetic beads. After cleavage a label can be introduced facilitating quantification/detection of the glycopeptides.

A proof of concept was prepared with a synthetic NeuAc-GalNAc-peptide. The sialic acid was mild oxidized using $\mathrm{NalO}_{4}$. The aldehyde was reacted with the modified beads in acetate buffer.

The beads were washed with PBS buffer for storage and after days the peptide was released and analyzed via MS.

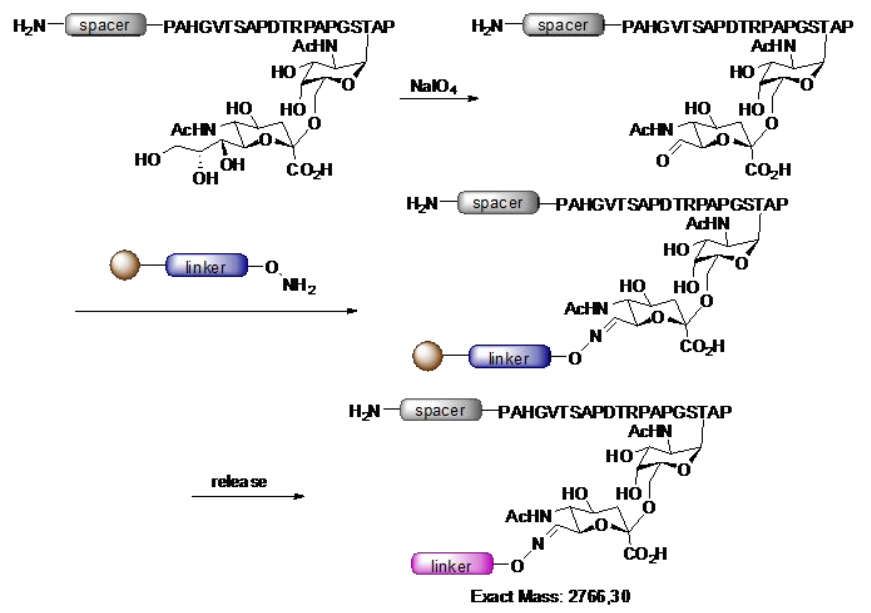

Scheme 1. Peptide labeling.

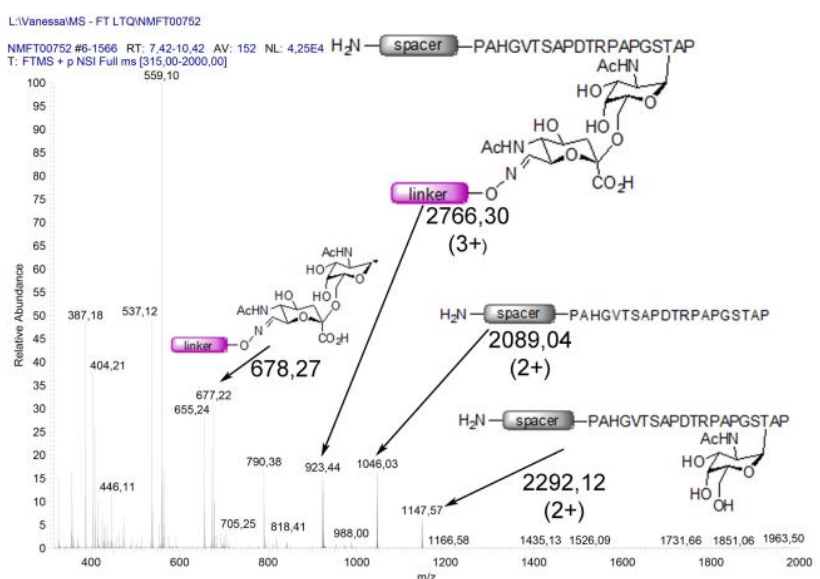

Figure 1. Peptide MS-spectra.

\section{CONCLUSION}

Glycopeptide model was prepared and our proof peptide was recovered with success from the beads.

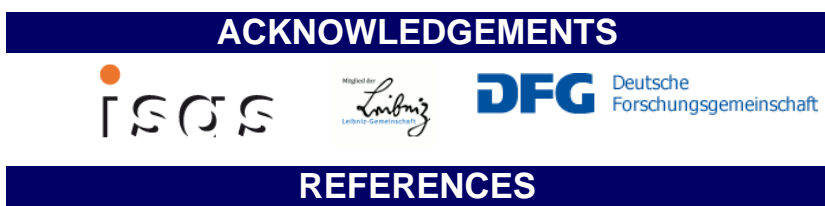

${ }^{1}$ Buskas, T.; Ingale, S.; Boons, G.-J. Glycobiology 2006, 16, 113.

${ }^{2}$ Lai, Z. W.; Nice, E. C.; Schilling O. Proteomic 2013, 13, 512.

3 a) Sletten, E.M.; Bertozzi, C.R. Angew. Chem. Int. Ed. 2009, 48, 6974; b) Nilsson, J.; Rüetschi, U.; Halim, A.; Hesse, C.; Carlsohn, E.; Brinkmalm, G.;

Larson, G. Nature Methods, 2009, 6, 809. 